\title{
Constitutional design: separation of financing and project decision
}

\section{Working Paper}

\section{Author(s):}

Gersbach, Hans; Hahn, Volker; Imhof, Stephan

Publication date:

2009-05

Permanent link:

https://doi.org/10.3929/ethz-a-005859341

Rights / license:

In Copyright - Non-Commercial Use Permitted

Originally published in:

Economics Working Paper Series 09/109 


\section{CER-ETH - Center of Economic Research at ETH Zurich}

\section{Constitutional Design: \\ Separation of Financing and Project Decision}

Hans Gersbach, Volker Hahn and Stephan Imhof

Working Paper 09/109

May 2009

\section{Economics Working Paper Series}

\section{EH}

Eidgenössische Technische Hochschule Zürich Swiss Federal Institute of Technology Zurich 


\title{
Constitutional Design: Separation of Financing and Project Decision*
}

\author{
Hans Gersbach \\ CER-ETH Center of Economic Research \\ at ETH Zurich and CEPR \\ 8032 Zurich, Switzerland \\ hgersbach@ethz.ch
}

\author{
Volker Hahn \\ CER-ETH Center of Economic Research \\ at ETH Zurich \\ 8032 Zurich, Switzerland \\ vhahn@ethz.ch
}

\author{
Stephan Imhof \\ CER-ETH Center of Economic Research \\ at ETH Zurich \\ 8032 Zurich, Switzerland \\ stimhof@ethz.ch
}

First Version: November 2007

This Version: May 2009

\begin{abstract}
We examine the provision of public projects under separate tax and subsidy rules. We find that tax rules separated from project cum subsidy decisions exhibit several advantages when incentive problems of the agenda-setter are taken into account. In particular, tax rules may prevent the proposal of inefficient projects which benefit only a small lobby group. We propose "redistribution efficiency" as a socially desirable property of proposals and find that tax rules always guarantee redistribution efficiency. We show that rules on subsidies combined with discretion regarding taxes always yield socially inferior proposals. Finally, tax rules induce the agenda-setter to look for potential improvements of public projects.
\end{abstract}

Keywords: constitutional design, provision of public projects, voting, taxes and subsidies

JEL Classification: D72, H40

\footnotetext{
${ }^{*}$ We would like to thank Felix Bierbrauer, Martin Hellwig, Matthew Jackson, Matthias Lang, Massimo Morelli, and participants at the workshop "Public Goods", Zurich 2009 for valuable comments and suggestions.
} 


\section{Introduction}

Democratic societies are characterized by separation between the financing of government expenditures and public project cum subsidy decisions. The financing of government expenditures is determined by detailed tax laws. Separately, legislatures decide on public projects and associated subsidy payments to members of the polity. Usually there are no legal constraints on the chosen subsidy scheme for a specific project.

This separation can be illustrated by various examples. Consider first public housing programs. The government builds houses and subsidizes rents so that a particular group in society particularly benefits from the program. The program is financed through tax revenues raised according to a separate tax law and independently of the specific project. Second, the government may invest in public transportation and simultaneously subsidize tickets for specific subgroups, such as the elderly. The subsidies are taken from government revenues generated by direct and indirect taxation. Third, a government may decide to foster growth in a particular region by investing in public infrastructure while simultaneously subsidizing entrepreneurs willing to move to this region. Again, financing occurs through tax revenues, independently of who benefits from the specific project.

In this paper we provide a political-economy rationale for the separation between the financing of government expenditures and public project cum subsidy decisions. We also explore the limitations of this widespread procedure.

In standard models of mechanism design, tying the benevolent mechanism designer's hands by imposing restrictions on possible tax schemes can never be welfare improving. ${ }^{1}$ By contrast, our paper assumes an agenda-setter who pursues his own interests. This opens up a potential role for restrictions on taxes to improve welfare.

We consider a society with a continuum of citizens where an agenda-setter can make a proposal about the adoption of a public project and the distribution of taxes and subsidies. The proposal is adopted if it is supported by a majority of voters. Our model involves three incentive problems. First, the agenda-setter may want to provide a public project if it is beneficial to her, although the project may be undesirable

\footnotetext{
${ }^{1}$ For a survey of the literature on mechanism design see Jackson (2001).
} 
from a utilitarian perspective. Second, the agenda-setter may want to raise more taxes than necessary in order to pay out subsidies to herself or to other citizens. Third, the agenda-setter may not want to look for the most efficient variant of the public project. We investigate the types of rules that are suitable for limiting the distortions arising from these incentive problems. We obtain five major findings.

First, we find that tax rules may prevent the agenda-setter from securing the necessary majority of voters for socially inefficient projects that benefit only a small lobby group. By contrast, the absence of tax rules enables the proposer to enforce any project irrespective of its characteristics.

Second, tax rules reduce wasteful subsidies to a minimum, i.e. only redistributionefficient proposals are made. The intuition for this finding is that under tax rules a large amount of total subsidies also implies high taxes for the agenda-setter.

Third, a constitution with rules on both taxes and subsidies is robust to counterproposals, whereas the other constitutions under consideration are prone to cycles of project adoption and project reversal.

Fourth, we find that in combination with arbitrary taxation subsidy rules yield high welfare losses. Such a constitution is inferior to one with no rules on taxes and subsidies. Fifth, an additional rationale for tax rules materializes when project characteristics are endogenous. We show that only constitutions involving tax rules induce the agendasetter to enhance project efficiency.

Overall, our paper provides a rationale suggesting that if incentive problems for the agenda-setter are taken into account decisions on projects cum subsidies should be separated from decisions on tax rules. Tax rules encoded in tax laws separated from public project cum subsidy decisions have several advantages over a scheme in which financing, project decision, and subsidies are jointly put to a vote.

The paper is organized as follows: We review the related literature in Section 2. Section 3 develops the basic framework. Sections 4-7 examine the outcomes for constitutions that differ with respect to their rules on taxes and subsidies. In Section 8 we examine the welfare implications of different constitutions. Section 9 discusses socially optimal constitutional rules for different categories of projects. We analyze endogenous project design in Section 10, and Section 11 concludes. 


\section{Relation to the Literature}

There are no other studies inquiring why a polity may adopt strict tax rules but allow flexibility in using subsidies in public-project provision. Our paper is a contribution to constructive constitutional economics, as outlined in the seminal study by Buchanan and Tullock (1962). Using the veil-of-ignorance device (see Rawls (1971)), Buchanan and Tullock (1962) have examined the costs and benefits of majority rules. ${ }^{2}$ Aghion and Bolton (2003) have refined and expanded this approach. When a society faces deadweight costs of redistribution, simple or supermajority rules are preferred to unanimity in order to overcome vested interests. ${ }^{3}$ Gersbach (2004) and Gersbach (2009) show that increasingly sophisticated agenda and decision rules further improve the efficiency of public-project provision when all admissible rules have to fulfill the liberal democracy constraint.

In this paper we focus on the efficiency properties of the simple or supermajority rule when it is coupled with tax or subsidy rules. Our main insight is that tax rules exhibit a variety of advantages and can rationalize the separation of taxation from public-project provision and subsidies. ${ }^{4}$

There is an extensive body of literature on optimal mechanisms for providing public goods when income taxes are a source of public-goods finance. ${ }^{5}$ Hellwig (2004) shows that when both income taxation and public-sector pricing are plagued by incentive considerations it is desirable to use a combination of income taxation and admission fees to finance public goods. We disregard incentive considerations at the citizen level and assume that financing is achieved by a simple, possibly personalized, lump-sum tax scheme. Our focus is on the incentive problem of the agenda-setter. ${ }^{6}$

\footnotetext{
${ }^{2}$ Closely related ideas have been developed by Rae (1969) and Taylor (1969).

${ }^{3}$ In a complete contract framework, Romer and Rosenthal (1983) have established that a unanimity rule may implement the full-information efficient solution when payoffs are private information but no deadweight costs of transfers arise.

${ }^{4}$ In our model, subsidies can be used to ensure the majority necessary for the adoption of a proposal and thus represent the institutionalized way of forming majorities in advanced democracies. There is an extensive body of literature on vote buying (see Groseclose and Snyder (1996) and Dekel et al. (2008), among others), where agenda-setters buy votes by using a stock of wealth.

${ }^{5}$ From Güth and Hellwig (1986), Rob (1989), Mailath and Postlewaite (1990), and Hellwig (2003) we know that in large economies it is impossible to obtain efficient outcomes in public-good provision if participation constraints have to be respected.

${ }^{6}$ Bierbrauer (2009) shows that coercion is desirable for the financing of public goods if those goods are provided by a malevolent leviathan. We take it as given that the government can impose taxes.
} 


\section{Model}

\section{$3.1 \quad$ Set-up}

We consider a society facing the standard problem of public-project provision and financing. Citizens are indexed by $j$ and are uniformly arranged on the unit interval. The provision of a public project yields utility $v_{j} \forall j \in[0,1]$ and involves per-capita costs $k \geq 0$. For simplicity of exposition, we assume $v_{j} \in\left\{V_{w}, V_{l}\right\}$ with $V_{w} \geq 0$ and $V_{w}>V_{l}$. Accordingly, we refer to individuals obtaining $V_{w}$ from the public project provision as "project winners" and to individuals receiving benefits $V_{l}$ as "project losers." Without loss of generality, we assume the project winners to be located on the interval $[0, p]$ and the project losers to be located on the interval $] p, 1]$.

One particular individual can make a proposal $\pi$, which comprises a tax and a subsidy distribution as well as the project decision. There are different ways of modeling which citizen has the right to set the agenda. We adopt the view that in a democracy it is impossible to deter beneficiaries of public projects from making proposals. ${ }^{7}$ Hence we directly assume that the agenda-setter is a project winner. Without loss of generality, we assume that the agenda-setter is $j=0$.

Subsidies and taxes are constrained to be non-negative. Moreover, there is some maximal level of subsidies denoted by $\hat{s}$ with $\hat{s} \geq V_{w}-V_{l}{ }^{8}$ Let $\mathbb{S}$ be the set of all non-negative Lebesgue-measurable functions on the unit interval that do not exceed $\hat{s}$. Thus each subsidy scheme involved by a proposal $\pi$ is a function $s(\pi) \in \mathbb{S}$. Accordingly, let $\mathbb{T}$ be the set of all non-negative Lebesgue-measurable functions on the unit interval. Then each tax scheme can be written as a function $t(\pi) \in \mathbb{T}$. Moreover, we use $g(\pi) \in\{0,1\}$ as a variable indicating whether the project will be adopted $(g(\pi)=1)$ or not $(g(\pi)=0)$ according to proposal $\pi .^{9}$

We consider distortionary taxes, i.e. for each unit of taxes that is paid by a particular individual, only a fraction $1 /(1+\lambda)(\lambda>0)$ can be used to finance the project or

\footnotetext{
${ }^{7}$ Another approach commonly applied is random selection of agenda-setters (see Gersbach (2009)).

${ }^{8}$ The assumption $\hat{s} \geq V_{w}-V_{l}$ simplifies the exposition, but does not qualitatively affect our findings.

${ }^{9}$ Lizzeri and Persico (2001) consider a model where the agenda-setter must choose between redistribution and public-project provision. In our model, it is possible to combine public projects with redistribution.
} 
subsidies. There are various interpretations of $\lambda$. It may represent resources used for collecting and transferring funds from citizens to the state. The deadweight costs $\lambda$ may also represent the disincentive to work if wages are taxed. The assumption of linear deadweight costs can be justified by the relationship between taxes paid for the public project and individual income, the former being sufficiently smaller than the latter. Now the society's budget constraint is

$$
(1+\lambda)[g(\pi) k+S(\pi)]=T(\pi)
$$

where we have introduced total subsidies $S(\pi):=\int_{0}^{1} s_{j}(\pi) d j$ and total tax revenues $T(\pi):=\int_{0}^{1} t_{j}(\pi) d j$ implied by proposal $\pi$. We assume $V_{w}-(1+\lambda) k>0$ and $V_{l}-(1+$ $\lambda) k<0$.

Now we are in a position to give a formal description of the general set of possible proposals.

$$
\Pi:=\{\pi \in\{0,1\} \times \mathbb{S} \times \mathbb{T} \mid(1+\lambda)[g(\pi) k+S(\pi)]=T(\pi)\} .
$$

\subsection{Constitutions}

We adopt the standard "veil of ignorance" procedure for constitutional design. The social choice problem is reduced to a two-period setting. The first period is the constitutional period and the second the legislative period. In the constitutional period all citizens are assumed to be identical and do not know whether they will be project winners or project losers. Moreover, the project's parameters $V_{w}, V_{l}, k$, and $p$ are not known with certainty. Citizens design a constitution or an incomplete social contract governing the supply of public goods, given a commonly known distribution of the project parameters. $^{10}$

Under the incomplete contract perspective, rules cannot depend on project characteristics, as those characteristics are not verifiable in court. Consequently, the rules that can be adopted in the constitutional period are those constraining the tax and subsidy schemes the agenda-setter is allowed to use. In this paper we adopt the perspective that in the constitutional period the decision rule that will be used later is given. In

\footnotetext{
${ }^{10}$ Incomplete social contracts have been studied by Aghion and Bolton (2003) and Gersbach (2009), among others.
} 
particular, we assume that a proposal will be adopted if it receives at least a fraction $m$ of all votes. The only assumption we make is that $\frac{1}{2} \leq m<\min \left\{\frac{1}{1+\lambda}+p, 1\right\} .{ }^{11}$ As we show later this assumption will significantly simplify our analysis.

In our model a constitution is simply a set of rules that constrain the set of proposals the agenda-setter can make. Accordingly, $\Pi$ represents a particular constitution, namely one without any further rules. In the course of the article we will consider less discretionary constitutions and impose rules on tax and/or on subsidy distribution. These constitutions represent subsets of $\Pi$.

It will be useful to define the set of all possible projects. It comprises all quadruples $\left(V_{w}, V_{l}, k, p\right)$ that satisfy the assumptions we have introduced so far. Formally, it is given by

$$
\begin{aligned}
\mathcal{P} & :=\left\{\left(V_{w}, V_{l}, k, p\right) \in \mathbb{R}^{+} \times \mathbb{R} \times \mathbb{R}^{+} \times\right] 0 ; 1[: \\
& \left.p>m-1 /(1+\lambda), V_{w}>(1+\lambda) k, V_{l}<(1+\lambda) k, \hat{s} \geq V_{w}+V_{l}\right\},
\end{aligned}
$$

where $\mathbb{R}$ and $\mathbb{R}^{+}$denote the sets of real numbers and non-negative real numbers respectively. The prior distribution of the project parameters can now be described by a joint probability density function on $\mathcal{P}$. At this stage, we do not specify a particular form for this density function.

\subsection{The legislative period}

In the legislative period, each individual observes $v_{j}$ and $k$, and all individual valuations become common knowledge. ${ }^{12}$ The agenda-setter makes a proposal that must obey the constitutional rules, otherwise the proposal is declared to be unconstitutional and the status quo prevails.

If proposal $\pi$ is adopted, the utility of individual $j \in[0 ; 1]$ will be b $^{13}$

$$
u_{j}(\pi)=g(\pi) v_{j}+s_{j}(\pi)-t_{j}(\pi) .
$$

\footnotetext{
${ }^{11}$ Plausible estimates of $\lambda$ lie between 0.2 and 0.5 (see Stuart (1984), Ballard et al. (1985), and Browning (1987)). For these estimates and the simple majority rule $(m=1 / 2)$ the assumption $1 / 2 \leq m<\min \{1 /(1+\lambda)+p, 1\}$ is always fulfilled.

${ }^{12} \mathrm{~A} n$ interesting variant of our model would involve citizens having private information about their types $v_{j} \in\left\{V_{w}, V_{l}\right\}$, while the value of $p$ is commonly known. This variant leads to results similar to those in this paper. A formal analysis of this case is available upon request.

${ }^{13}$ We assume that the income of individuals is sufficient to pay taxes under any proposal considered in the paper.
} 
We assume that each individual will vote in favor of the proposal if and only if $u_{j}(\pi) \geq$ 0 . It will be useful to define the indicator function $I(\pi)$, which adopts a value of 1 if the proposal is implemented and of zero otherwise.

$$
I(\pi):= \begin{cases}1 & \text { if } u_{j}(\pi) \geq 0 \text { for at least a fraction } m \text { of voters } \\ 0 & \text { otherwise }\end{cases}
$$

Thus we can write the expected utility of individual $j$ as $U_{j}(\pi)=I(\pi) u_{j}(\pi)$ given proposal $\pi$ has been made. In addition, the utilitarian welfare measure for a particular proposal $\pi$ amounts to

$$
W(\pi):=I(\pi)\left[\left(p V_{w}+(1-p) V_{l}-k(1+\lambda)\right) g(\pi)-\lambda S(\pi)\right]
$$

For the sake of simplicity, we introduce the following tie-breaking rule: If the agendasetter is indifferent between several proposals, she will choose a proposal with the highest $u_{0}(\pi)$, i.e. a proposal that if implemented would yield the highest utility for her.

\subsection{Socially efficient solutions}

As a starting point it is instructive to consider socially optimal proposals. Consider a social planner who maximizes the utilitarian welfare measure by choosing and implementing a proposal $\pi \in \Pi$ for a given realization of the project parameters $V_{w}, V_{l}, k$, and $p$. It is obvious that the following lemma holds:

\section{Lemma 1}

A socially optimal proposal $\pi$ has the following characteristics:

$$
\begin{aligned}
& g(\pi)= \begin{cases}1 & \text { for } p V_{w}+(1-p) V_{l} \geq(1+\lambda) k \\
0 & \text { for } p V_{w}+(1-p) V_{l}<(1+\lambda) k\end{cases} \\
& S(\pi)=0 .
\end{aligned}
$$

In particular, the social planner will never choose a positive level of total subsidies because of the losses caused by distortionary taxation. We note that the socially optimal solution is not normally unique because for $g(\pi)=1$ the social planner is indifferent with respect to all possible tax schemes raising the revenues necessary to finance the project. 
If the project parameters $p, V_{w}, V_{l}$, and $k$ were verifiable, it would be straightforward to characterize a constitution guaranteeing the optimal level of welfare. However, we assume in the following that constitutional rules cannot depend on project characteristics, as even for perfectly observable costs and benefits of projects it is plausible that the project characteristics are not verifiable in court.

\subsection{Evaluation criteria}

In the following we establish several desirable properties of constitutions. For this purpose it will be useful to define the following concept:

\section{Definition 1}

For a given constitution $\widetilde{\Pi} \subseteq \Pi$, a proposal $\pi \in \widetilde{\Pi}$ with $I(\pi)=1$ is redistributionefficient if no $\pi^{\prime} \in \widetilde{\Pi}$ exists with $S\left(\pi^{\prime}\right)<S(\pi), g(\pi)=g\left(\pi^{\prime}\right)$ and $I\left(\pi^{\prime}\right)=1$. A proposal with $I(\pi)=0$ is always redistribution-efficient.

For example, we refer to a proposal $\pi$ that ensures the adoption of the project as redistribution-efficient if no alternative proposal exists that would guarantee the adoption of the project while involving strictly lower total subsidies. It is obvious that redistribution-efficiency is a desirable property of proposals, as it keeps wasteful redistribution to a minimum.

\section{Definition 2}

We refer to a constitution under which only redistribution-efficient proposals are made as a constitution satisfying GREP (guarantees redistribution-efficient proposals).

Now we turn to further desirable property of constitutions. While it is plausible that designing socially desirable projects is difficult, it may be much easier to conceive of socially harmful projects that benefit only a small lobby group. Thus one important feature of a constitution may be that it prevents the adoption of projects of this kind. To be more precise, we define the set of lobby projects $L P(\varepsilon) \subset \mathcal{P}$ for $\varepsilon<1 / 2$ as the set of all projects in $\mathcal{P}$ with parameters $V_{w}, V_{l}, k$, and $p$ such that $\left|V_{w}-k(1+\lambda)\right|<$ $\left|V_{l}-k(1+\lambda)\right|$ and $p \leq \varepsilon$. Note that condition $\left|V_{w}-k(1+\lambda)\right|<\left|V_{l}-k(1+\lambda)\right|$ can be interpreted as the net benefits of project winners being lower than the net losses of project losers. For $p<1 / 2$ this obviously implies $p V_{w}+(1-p) V_{l}<(1+\lambda) k$. 


\section{Definition 3}

A constitution satisfies the property of "protection against lobby projects" (henceforth $P A L P)$ if a value for $\varepsilon \in] 0 ; 1 / 2[$ exists such that all projects in the set $L P(\varepsilon)$ are never adopted in equilibrium.

As a consequence, citizens would agree on a constitution satisfying PALP under a veil of ignorance if sufficient significance were attached to bad lobby projects in the prior distribution of project characteristics.

The reversal of some projects, like the construction of public buildings or infrastructure, may be prohibitively costly compared to the benefits involved. But in other cases project reversal may be relatively easy. Examples are a reform of penal law or changes to the tax system. For these cases, we cannot rule out the eventuality of one of the project losers proposing to reverse the project after a proposal has been adopted. It is obvious that a sequence of project adoption, reversal, renewed project adoption, and so forth is not desirable. Thus we propose robustness against counter-proposals (henceforth $R A C P$ ) as another criterion for evaluating constitutions. More specifically, we assume that potentially reversible projects involve negligible costs $k$, i.e. $k=0$. So if the original project involves $p=p^{0}, V_{l}=V_{l}^{0}$, and $V_{w}=V_{w}^{0}$, reversal of the project can be characterized by $p=1-p^{0}, V_{l}=-V_{w}^{0}$, and $V_{w}=-V_{l}^{0}$.

In order to consider the reversal of projects, we have to specify a game involving a sequence of legislative stages. For simplicity of exposition we assume that agendasetters and voters are short-sighted when making a proposal or voting. For example, when a decision is to be taken, voters do not take into account the eventuality of the project being reversed in the future. This assumption does not qualitatively affect our results. To sum up, a potentially reversible project can be reversed if both itself and the reversal of the project can be adopted in an equilibrium of our basic model.

We are now in a position to define $R A C P$ as follows:

\section{Definition 4}

A constitution displays robustness against counter-proposals ( $R A C P$ ) if no potentially reversible project can be reversed by a respective counter-proposal. 
Obviously, it may be possible to rule out project reversal directly in the constitution. However, in a richer framework with project costs and benefits that are uncertain before implementation, such a constitutional rule may be disadvantageous as it eliminates the possibility to reverse projects that have turned out to be much less desirable than expected. Then constitutions displaying $R A C P$ may be desirable.

\section{Arbitrary Tax Code and Arbitrary Subsidy Scheme}

In our first scenario we impose no additional rules on taxes and subsidies, i.e. the agenda-setter can choose any proposal $\pi \in \Pi$.

\section{Proposition 1}

For constitution $\Pi$ the agenda-setter will always choose a proposal $\pi^{*}$ with $g\left(\pi^{*}\right)=1$, $t_{0}\left(\pi^{*}\right)=0, s_{0}\left(\pi^{*}\right)=\widehat{s}$, and $I\left(\pi^{*}\right)=1$.

\section{Proof}

The agenda-setter solves the following problem:

$$
\max _{\pi \in \Pi}\left\{\left(g(\pi) V_{w}+s_{0}(\pi)-t_{0}(\pi)\right) I(\pi)\right\} .
$$

It is obvious that $g(\pi)=1, t_{0}(\pi)=0$, and $s_{0}(\pi)=\widehat{s}$ guarantee maximum utility for the agenda-setter, provided that the proposal is actually adopted. Importantly, a proposal with $g(\pi)=1, t_{0}(\pi)=0$, and $s_{0}(\pi)=\widehat{s}$ that entails $I(\pi)=1$ always exists. For example, the agenda-setter can impose zero taxes on all individuals from the interval ] $0 ; m]$ and tax all individuals from the interval $] m, 1]$ identically to cover the costs for the project $k$ and the subsidies $S(\pi)$ that may be necessary to gain support from all members in $] 0 ; m]$.

Interestingly, we obtain the following lemma:

\section{Lemma 2}

For constitution $\Pi$ a proposal chosen by the agenda-setter may be redistributioninefficient. Therefore constitution $\Pi$ does not satisfy GREP.

\section{Proof}

The proof of this lemma is straightforward. Suppose a redistribution-efficient proposal 
$\pi^{*}$ exists that maximizes the agenda-setter's utility. It is obvious that for $\pi^{*}$ some project losers exist who receive no subsidies $\left(s_{j}\left(\pi^{*}\right)=0\right)$. Now we can modify $\pi^{*}$ by introducing positive subsidies for these individuals, which are financed by additional taxes for these very persons. The resulting proposal would also be adopted, but is clearly not redistribution-efficient. Thus for each redistribution-efficient proposal we can find a multitude of proposals that are not redistribution-efficient.

In addition, as all projects are adopted, it is obvious that the following lemma holds:

\section{Lemma 3}

Constitution $\Pi$ does not satisfy PALP.

Intuitively, the high degree of flexibility for the proposer enables her to adopt any project, independently of its characteristics. Hence socially detrimental lobby projects are always implemented.

Finally we note

\section{Lemma 4}

Constitution $\Pi$ does not satisfy $R A C P$.

As any project can be adopted by a suitable tax-subsidy scheme, it is obvious that any project can be reversed by a respective counter-proposal.

\section{Uniform Tax Code and Arbitrary Subsidy Scheme}

Now we impose the requirement that all individuals have to be treated identically with respect to taxation. Recall that individuals have the same income, so equal taxation is the only tax rule that is non-discriminatory. As a consequence, we consider the following constitution:

$$
\Pi_{T}:=\left\{\pi_{T} \in \Pi \mid t_{j}\left(\pi_{T}\right)=t_{i}\left(\pi_{T}\right) \quad \forall i, j \in[0,1]\right\} .
$$

Here the agenda-setter can only choose proposals from $\Pi_{T} \subset \Pi$. For notational convenience we define

$$
V_{w}^{*}:=(1+\lambda) \frac{k-(m-p) V_{l}}{1-(1+\lambda)(m-p)}
$$


Note that, for $p<m, V_{w}^{*}>0$ follows from the assumption $m<\min \left\{\frac{1}{1+\lambda}+p, 1\right\}$. It is straightforward to verify $V_{w}^{*}-V_{l}>0$, which follows from $V_{l}-(1+\lambda) k<0$.

\section{Proposition 2}

For constitution $\Pi_{T}$ a unique ${ }^{14}$ equilibrium proposal $\pi_{T}^{*}$ exists.

1. For $V_{w} \geq V_{w}^{*}$ and $p<m, \pi_{T}^{*}$ is given by $g\left(\pi_{T}^{*}\right)=1, t_{j}\left(\pi_{T}^{*}\right)=V_{w}^{*} \forall j \in[0,1]$, and

$$
s_{j}\left(\pi_{T}^{*}\right)= \begin{cases}\hat{s} & \text { for } j=0 \\ 0 & \text { for } j \in] 0, p] \\ V_{w}^{*}-V_{l} & \text { for } j \in] p, m] \\ 0 & \text { for } j \in] m, 1]\end{cases}
$$

2. For $V_{w}<V_{w}^{*}$ and $p<m, \pi_{T}^{*}$ is given by $g\left(\pi_{T}^{*}\right)=0, t_{j}\left(\pi_{T}^{*}\right)=0 \forall j \in[0,1]$, and

$$
s_{j}\left(\pi_{T}^{*}\right)= \begin{cases}\hat{s} & \text { for } j=0 \\ 0 & \text { for } j \in] 0,1] .\end{cases}
$$

3. For $p \geq m$, $\pi_{T}^{*}$ is given by $g\left(\pi_{T}^{*}\right)=1, t_{j}\left(\pi_{T}^{*}\right)=(1+\lambda) k \forall j \in[0,1]$, and

$$
s_{j}\left(\pi_{T}^{*}\right)= \begin{cases}\hat{s} & \text { for } j=0 \\ 0 & \text { for } j \in] 0,1] .\end{cases}
$$

The equilibrium proposal is always adopted.

The proof of Proposition 2 is given in Appendix A. According to Proposition 2, the agenda-setter will always choose the maximum level of subsidies for herself, which is plausible. In the following, we discuss the three cases mentioned in the proposition separately.

For $p \geq m$, the project winners alone can enforce the adoption of the project. As a consequence, a proposal will secure the necessary majority, even if it involves zero total subsidies, i.e. subsidies only to a group of Lebesgue measure zero.

For $p<m$, it is necessary to subsidize some project losers to induce them to accept the project. In the proof we show that $V_{w}^{*}$ represents the level of taxes that is necessary to finance these subsidies. For $V_{w} \geq V_{w}^{*}$, the project winners' gains $V_{w}$ from the project exceed this tax level. However, for $V_{w}<V_{w}^{*}$ the benefits of the project winners are so

\footnotetext{
${ }^{14}$ More precisely, the proposal is unique up to relabeling individuals and redistribution within masses of Lebesgue measure zero. We will use "unique" in this sense throughout the paper.
} 
low that they are not willing to finance the subsidies necessary to induce some project losers to support the proposal. Thus the agenda-setter will choose a proposal $\pi_{T}$ with $g\left(\pi_{T}\right)=0$.

It is important to note that the agenda-setter always chooses a redistribution-efficient proposal. A proposal with a higher level of total subsidies $S(\pi)$ would entail a higher level of taxes, which would be harmful to the agenda-setter. We summarize this observation in the following lemma:

\section{Lemma 5}

Constitution $\Pi_{T}$ satisfies $G R E P$, i.e. proposal $\pi_{T}^{*}$ is always redistribution-efficient.

In Appendix B we also show

\section{Lemma 6}

Constitution $\Pi_{T}$ satisfies $P A L P$.

We note that projects with $k=0, p<m$, and $V_{w} \geq V_{w}^{*}$ are susceptible to counterproposals. As a consequence, we obtain

\section{Lemma 7}

Constitution $\Pi_{T}$ violates $R A C P$.

\section{Arbitrary Tax Code and Uniform Subsidy Scheme}

Now we consider a constitution that allows for arbitrary tax schemes. However, we limit the subsidy schemes to those that treat all citizens identically. Hence we restrict our attention to the set of proposals $\Pi_{S} \subset \Pi$ with

$$
\Pi_{S}:=\left\{\pi_{S} \in \Pi \mid s_{j}\left(\pi_{S}\right)=s_{i}\left(\pi_{S}\right) \quad \forall i, j \in[0,1]\right\}
$$

For this case we obtain

\section{Proposition 3}

For constitution $\Pi_{S}$ each equilibrium proposal $\pi_{S}^{*}$ can be characterized by $g\left(\pi_{S}^{*}\right)=1$, $s_{j}\left(\pi_{S}^{*}\right)=\hat{s} \forall j \in[0,1], t_{0}\left(\pi_{S}^{*}\right)=0$, and $I\left(\pi_{S}^{*}\right)=1$. 


\section{Proof}

The agenda-setter solves the following problem:

$$
\left.\max _{\pi \in \Pi}\left\{g(\pi) V_{w}+s(\pi)-t_{0}(\pi)\right) I(\pi)\right\} .
$$

It is obvious that $t_{0}(\pi)=0, s(\pi)=\hat{s}, g(\pi)=1$ guarantee the highest possible payoff for the agenda-setter, provided that she can induce enough voters to support such a proposal. It is always possible to secure the necessary majority by taxing only the individuals from the interval $] m ; 1]$. In this case, non-taxed project winners will vote in favor of $\pi$ (as $V_{w}+\hat{s}>0$ ) as well as non-taxed project losers (as $V_{l}+\hat{s}>0$ ), which implies $I(\pi)=1$.

As each proposal under $\Pi_{S}$ involves the maximum amount of total subsidies $S(\pi)=\widehat{s}$ and a proposal would also be accepted for slightly lower total subsidies, we obtain

\section{Lemma 8}

Under constitution $\Pi_{S}$ the equilibrium proposal is never redistribution-efficient. As a consequence, $\Pi_{S}$ violates $G R E P$.

As constitution $\Pi_{S}$ enables the proposer to implement all projects, even very poor ones, it is obvious that

\section{Lemma 9}

Constitution $\Pi_{S}$ does not satisfy PALP.

Additionally, it is straightforward to see

\section{Lemma 10}

Constitution $\Pi_{S}$ does not satisfy $R A C P$.

\section{Uniform Tax Code and Uniform Subsidy Scheme}

Finally we consider a constitution that stipulates that all citizens be treated equally with respect to subsidies and taxes. Hence the set of feasible proposals reduces to $\Pi_{S T} \subset \Pi$, where

$$
\Pi_{S T}:=\Pi_{T} \cap \Pi_{S}
$$

For this constitution we obtain 


\section{Proposition 4}

For constitution $\Pi_{S T}$ the equilibrium proposal $\pi_{S T}^{*}$ is unique with $s_{j}\left(\pi_{S T}^{*}\right)=0, t_{j}\left(\pi_{S T}^{*}\right)=$ $(1+\lambda) k \forall j \in[0,1]$, and $g\left(\pi_{S T}^{*}\right)=1$. For this proposal $I\left(\pi_{S T}^{*}\right)=1$ iff $p \leq m$.

\section{Proof}

The agenda-setter solves the following problem:

$$
\max _{\pi \in \Pi}\left\{\left(g(\pi)\left(V_{w}-(1+\lambda) k\right)+s(\pi)-(1+\lambda) s(\pi)\right) I(\pi)\right\} .
$$

Under constitution $\Pi_{S T}$, introducing subsidies is not worthwhile for the agenda-setter as the taxes necessary to finance them are always higher. Thus a positive level of subsidies makes the proposal less attractive to all citizens, including the agenda-setter herself. As the agenda-setter always prefers project adoption, she will always propose implementing the project.

We note that, for $p \geq m$, the project winners are sufficiently numerous to enforce the project. For $p<m$ the project losers can block the project.

Because the proposal never involves subsidies, we can conclude

\section{Lemma 11}

Proposal $\pi_{S T}^{*}$ is always redistribution-efficient. Thus $\Pi_{S T}$ satisfies GREP.

Our finding that projects with $p<m$ are never adopted immediately implies

\section{Lemma 12}

Constitution $\Pi_{S T}$ satisfies $P A L P$.

Interestingly, under constitution $\Pi_{S T}$ a project will be adopted if and only if $p>m$, which immediately implies

\section{Lemma 13}

Constitution $\Pi_{S T}$ satisfies $R A C P$.

\section{Welfare Comparison}

In this section we compare social welfare. In Appendix $\mathrm{C}$ we derive the expressions for welfare for each constitution under a specific realization of project parameters $V_{w}, V_{l}$, $k$, and $p$. 
For constitution $\Pi$ welfare cannot be pinned down exactly, because a multitude of redistribution-inefficient proposals exist in addition to the redistribution-efficient proposals. However, it is possible to compute an upper boundary for welfare by computing welfare for redistribution-efficient proposals. In addition, the proposal with the highest possible level of total subsidies $\widehat{s}$ yields a lower boundary for welfare under $\Pi$.

$$
\begin{aligned}
& W\left(\pi^{*}\right) \leq p V_{w}+(1-p) V_{l}-(1+\lambda) k- \begin{cases}\lambda(m-p) \max \left\{0,-V_{l}\right\} & \text { if } p<m \\
0 & \text { if } p \geq m\end{cases} \\
& W\left(\pi^{*}\right) \geq p V_{w}+(1-p) V_{l}-(1+\lambda) k-\lambda \hat{s}
\end{aligned}
$$

Under constitution $\Pi_{T}$ the project is adopted if $p \geq m$ or if $p<m$ and $V_{w} \geq V_{w}^{*}$. Subsidies are only paid in the latter case. Hence the utilitarian welfare measure amounts to

$$
\begin{aligned}
W\left(\pi_{T}^{*}\right) & \begin{cases}p V_{w}+(1-p) V_{l}-(1+\lambda) k-\lambda(m-p)\left(V_{w}^{*}-V_{l}\right) & \text { if } p<m \text { and } V_{w} \geq V_{w}^{*} \\
0 & \text { if } p<m \text { and } V_{w}<V_{w}^{*} \\
p V_{w}+(1-p) V_{l}-(1+\lambda) k & \text { if } p \geq m .\end{cases}
\end{aligned}
$$

Under constitution $\Pi_{S}$ the project will always be adopted. Moreover, the agenda-setter will choose the maximum level of subsidies for herself and, because of the uniform subsidy rule, for all other citizens as well.

$$
W\left(\pi_{S}^{*}\right)=p V_{w}+(1-p) V_{l}-(1+\lambda) k-\lambda \hat{s}
$$

Under constitution $\Pi_{S T}$ no subsidies occur. Thus the project will be adopted if and only if it is beneficial to a majority.

$$
W\left(\pi_{S T}^{*}\right)= \begin{cases}0 & \text { if } p<m \\ p V_{w}+(1-p) V_{l}-(1+\lambda) k & \text { if } p \geq m .\end{cases}
$$

We note that constitutions $\Pi$ and $\Pi_{S}$ both yield project adoption for any admissible combination of the exogenous variables. However, $\Pi_{S}$ entails a higher level of total subsidies in general. As a consequence, constitution $\Pi_{S}$ is inferior to constitution $\Pi$ and thus never represents the socially optimal constitution. Intuitively, the desire of the agenda-setter to receive high subsidies together with the rule that all other citizens are also required to receive the same level of subsidies induces excessive redistribution under $\Pi_{S}$. Consequently, $\Pi_{S}$ would never be adopted under a veil of ignorance. 
For the other three constitutions $\Pi, \Pi_{T}$, and $\Pi_{S T}$ no general ranking with respect to welfare can be established that would hold for all admissible values of the exogenous variables. Which one of these would be selected would depend on the distribution of project parameters $V_{w}, V_{l}, k$, and $p$ in general.

We can rank constitutions $\Pi, \Pi_{T}$, and $\Pi_{S T}$ according to their degree of restrictiveness, with $\Pi$ the least restrictive and $\Pi_{S T}$ the most restrictive constitution. Note that the less restrictive the constitution is, the larger the set of parameter values will be for which the project is adopted. This is intuitive, as less restrictive constitutions grant the agenda-setter higher flexibility in designing a proposal that will secure the majority of votes. In particular, the least restrictive constitution $\Pi$ yields project adoption for any combination of parameters. The most restrictive constitution $\Pi_{S T}$ entails project adoption for $p \geq m$ only.

The most restrictive constitution $\Pi_{S T}$ has the advantage of eliminating any redistribution activity. However, for some parameter constellations this may involve costs, as projects are never adopted if $p<m$, although they may be socially desirable.

\section{Examples}

In the following we consider the implications of our model for different categories of projects. Two arguments support this approach. First, it may be known at the constitutional stage that a specific class of projects presents the major challenge facing the polity. Second, and perhaps more importantly, while it may not be possible to write constitutional rules dependent on project characteristics, it is plausible for different constitutional rules to be designed for different categories of projects. Project categories are likely to be verifiable, while the exact project parameters $V_{l}, V_{w}, p$, and $k$ are not. Accordingly, in the following we examine the optimal constitutional rules for different project categories. First we focus on the case of economic reform projects, then we examine locally beneficial projects. 


\subsection{Economic reforms}

We focus here on the special case of economic reforms, which represent a subset of $\mathcal{P}$. One important characteristic of economic reforms, such as labor-market reforms or product-market reforms leading to more intense competition, is that they are unlikely to involve substantial direct costs $k$. Thus we set $k=0$. Moreover, it is plausible to assume that economic reforms will differ in the effect they have on small interest groups and the large majority of the population. More specifically, we distinguish between socially beneficial economic reforms and socially detrimental reforms.

Socially beneficial reforms, such as the liberalization of the agricultural sector, are harmful to a small interest group, i.e. those working in this sector. However, they are beneficial to the rest of society as they stand to gain from lower prices or lower subsidies, which in turn imply tower taxes. For this class of reforms we assume that $p$ is larger than $m$ and that the total benefits are positive, i.e. $p V_{w}+(1-p) V_{l}>0$.

Socially detrimental reforms, like measures leading to lower competition in a specific sector, benefit only a small interest group, for example the shareholders of the firms in the specified sector. As a consequence, for these reforms $p<m$ and $p V_{w}+(1-p) V_{l}<0$ hold.

Interestingly, for economic reforms constitution $\Pi_{S T}$ will always implement the firstbest. All socially desirable projects are adopted, and socially harmful projects are never implemented. Moreover, there are no losses from redistribution. We summarize this finding in the following proposition:

\section{Proposition 5}

Constitution $\Pi_{S T}$ always leads to the first-best outcome.

We note that constitutions $\Pi$ and $\Pi_{S}$ are definitely inferior to $\Pi_{S T}$, as under the first two constitutions all reforms are adopted, including the socially detrimental ones. Constitution $\Pi_{T}$ may only lead to a welfare level identical to the one implied by constitution $\Pi_{S T}$ if $V_{w}<V_{w}^{*}$ holds for all socially detrimental reforms. Unless this is the case, $\Pi_{T}$ is strictly inferior to $\Pi_{S T}$ from an aggregate welfare perspective.

Hence, as far as economic reforms are concerned, highly restrictive rules maximize citizen utility from an ex-ante perspective under a veil of ignorance. 


\subsection{Locally beneficial projects}

Next we study the case of locally beneficial projects, such as hospitals, bridges, kindergartens, or theaters. These projects yield benefits to some of the citizens who live in the vicinity, but largely leave the utility for the majority of citizens unchanged. Accordingly, we assume $p<1-m$ and $V_{l}=0$. For simplicity we assume in the following that costs $k$ are uniformly distributed on the interval $[0 ; \bar{k}]$ and that $V_{w}$ and $p$ are drawn from a degenerate distribution. In Appendix D we show

\section{Proposition 6}

For locally beneficial projects there exists a critical value of $\bar{k}$, denoted by $\hat{k}$, such that

1. if $\bar{k}<\hat{k}$, then citizens will prefer $\Pi_{T}$ to $\Pi_{S T}$ from an ex-ante perspective;

2. if $\bar{k}>\hat{k}$, then citizens will prefer $\Pi_{S T}$ to $\Pi_{T}$ from an ex-ante perspective;

3. if $\bar{k}=\hat{k}$, then citizens will be indifferent with respect to $\Pi_{S T}$ and $\Pi_{T}$ from an ex-ante perspective.

To sum up, whether citizens would choose $\Pi_{S T}$ or $\Pi_{T}$ under a veil of ignorance depends on the distribution of the project's costs. If expected project costs are low, which corresponds to a low value of $\bar{k}$, then citizens will prefer $\Pi_{T}$ because this constitution will enable some projects to be adopted. However, it also involves losses due to the taxes that need to be levied in order to subsidize some of the project losers. Conversely, for high expected costs ( or high $\bar{k}$ ) citizens would prefer $\Pi_{S T}$, as this constitution eliminates the implementation of locally beneficial projects completely.

\section{Endogenous Project Characteristics}

So far, we have discussed which proposal will be chosen by the agenda-setter for given characteristics of the project. However, it seems reasonable to assume that the project parameters $V_{w}, V_{l}, k$, and $p$ are not exogenously given, but can be influenced by the agenda-setter to some extent. While it is plausible to assume that the proposer will attempt to design a project with high levels of $V_{w}$, which is to her own benefit, the interesting question arises as to the circumstances under which she may also affect project parameters $k, p$ and $V_{l}$ in a desirable way. An improvement of the project 
along these lines does not make the project more valuable to the agenda-setter directly. Instead, it increases its benefits for other citizens.

More specifically, we assume that the agenda-setter can exert effort before she makes the proposal. This effort creates costs $c>0$ for her. These costs are assumed to be so small that they have no bearing on welfare. We consider three different scenarios:

1. Improvement of the project for project losers:

$$
V_{l}= \begin{cases}\underline{V_{l}} & \text { if the agenda-setter does not exert effort } \\ \overline{V_{l}}>\underline{V_{l}} & \text { if the agenda-setter exerts effort. }\end{cases}
$$

2. Increase of the fraction of project winners:

$$
p= \begin{cases}\underline{p} & \text { if the agenda-setter does not exert effort } \\ \bar{p}>\underline{p} & \text { if the agenda-setter exerts effort. }\end{cases}
$$

3. Reduction of the project's costs:

$$
k= \begin{cases}\bar{k} & \text { if the agenda-setter does not exert effort } \\ \underline{k}<\bar{k} & \text { if the agenda-setter exerts effort. }\end{cases}
$$

In Appendix E we show

\section{Proposition 7}

1. Under constitutions $\Pi$ and $\Pi_{S}$ the agenda-setter has no incentive to enhance the project under all three scenarios.

2. Under constitution $\Pi_{S T}$ the agenda-setter may enhance the project by increasing $p$ and by decreasing project costs $k$. She will never improve $V_{l}$.

3. Under constitution $\Pi_{T}$ the agenda-setter may enhance the project under all scenarios.

Under constitutions $\Pi$ and $\Pi_{S}$ the agenda-setter can always achieve project adoption and does not pay any taxes under her equilibrium proposal. Consequently, her utility does not depend on parameters $V_{l}, p$, and $k$. Thus there are no incentives to incur the costs necessary for the improvement of the project under all scenarios. Similarly, the agenda-setter would never facilitate an increase in $V_{l}$ under constitution $\Pi_{S T}$. Exerting 
effort does not reduce taxes for her, nor does it increase the likelihood of the project being adopted.

There are, however, several cases where the agenda-setter may profit from exerting effort. This applies to constitutions involving tax rules, i.e. for $\Pi_{T}$ and $\Pi_{S T}$. Tax rules may induce agenda-setters to exert effort for two reasons. First, exerting effort may secure the adoption of a project that would otherwise be rejected. For example, if $\underline{p}<m$ and $\bar{p} \geq m$, exerting effort to increase $p$ will be optimal for the agenda-setter for sufficiently small $c$ under constitution $\Pi_{S T}$. Second, the agenda-setter may want to improve the project, as this lowers the subsidies necessary to gain support for the proposal, which in turn lowers her tax burden.

\section{Conclusions}

In this paper we have examined four constitutions with different restrictions on taxes and subsidies. We have shown that a constitution that imposes only the restriction of identical treatment with respect to subsidies is always inferior to a constitution that imposes no restrictions on taxes and subsidies. Thus constitution $\Pi_{S}$ would never be chosen at the constitutional stage.

Moreover, we have identified four advantages of tax rules. First, they always lead to redistribution-efficient proposals. As the agenda-setter has to pay the same amount of taxes as any other citizen, she avoids excessive subsidies. Second, tax rules may induce the agenda-setter to exert effort in order to improve the project. Exerting effort may reduce the subsidies required to enlist the support of sufficiently many voters, which also reduces taxes for the agenda-setter. Moreover, under tax rules the likelihood of project adoption is higher for more favorable projects. Third, constitutions without tax rules grant a high degree of flexibility to the agenda-setter, which enables her to gain support for any project, irrespective of its character. By contrast, constitutions with tax rules prevent the adoption of extremely bad projects that benefit only a small minority $p$, involve high costs $k$, and bring low benefits $V_{l}$ for losers. Fourth, a constitution with rules both on taxes and subsidies displays the desirable feature of robustness against counter-proposals. To sum up, our paper provides a rationale for the observation that decisions on project cum subsidies are usually made independently of decisions on rules that determine how government expenditures are financed. 


\section{A Proof of Proposition 2}

Step 1: Uniform taxes imply the following problem for the agenda-setter:

$$
\max _{\pi \in \Pi}\left\{\left(g(\pi)\left(V_{w}-(1+\lambda) k\right)+s_{0}(\pi)-(1+\lambda) S(\pi)\right) I(\pi)\right\}
$$

Recall that $u_{0}(\pi)=g(\pi)\left(V_{w}-(1+\lambda) k\right)+s_{0}(\pi)-(1+\lambda) S(\pi)$.

Step 2: We first construct the optimal proposal for the agenda-setter when the project is not proposed. We denote this proposal by $\tilde{\pi}$.

We claim that $\tilde{\pi}$ is given by $g(\tilde{\pi})=0, t_{j}(\tilde{\pi})=0, \forall j \in[0,1]$ and

$$
s_{j}(\tilde{\pi})= \begin{cases}\hat{s} & \text { for } j=0 \\ 0 & \text { for } j \in] 0,1] .\end{cases}
$$

To prove our claim, we first argue that, in equilibrium, proposal $\tilde{\pi}$ will be adopted (i.e. $I(\tilde{\pi})=1)$ as $u_{j}(\tilde{\pi}) \geq 0, \forall j \in[0,1]$.

Second, any other proposal would yield a smaller $u_{0}(\pi)$. Hence proposal $\tilde{\pi} \max -$ imizes $u_{0}(\pi) I(\pi)$ under the restriction that $g(\pi)=0$.

Step 3: We now consider proposals when the project is proposed. In contrast to Step 2, there is no unique proposal for all distributions of parameters $p, m, V_{w}, V_{l}$ and $k$. We therefore have to distinguish several cases.

Step 4: Consider the case $p \geq m$.

We claim that the optimal proposal for the agenda-setter is given by $g(\pi)=1$, $t_{j}(\pi)=(1+\lambda) k, \forall j \in[0,1]$ and $s_{j}(\pi)=s_{j}(\tilde{\pi})$.

To prove the claim, we first argue that, in equilibrium, proposal $\pi$ will be adopted (i.e. $I(\pi)=1$ ) as $u_{j}(\pi)>0, \forall j \in[0, p]$.

Second, any other proposal would yield a smaller $u_{0}(\pi)$. Hence proposal $\pi$ maximizes $U_{0}(\pi)=u_{0}(\pi) I(\pi)$ under the restriction that $g(\pi)=1$ and $p \geq m$.

Step 5: For the agenda-setter, proposal $\pi$ is preferable to proposal $\tilde{\pi}$, as

$$
U_{0}(\tilde{\pi})=\hat{s}<\hat{s}+V_{w}-(1+\lambda) k=U_{0}(\pi) .
$$

Hence, in case $p \geq m$, proposal $\pi$ will be implemented. 
Step 6: Consider the case $p<m$ and $V_{w} \geq V_{w}^{*}$.

We claim that the optimal proposal for the agenda-setter is given by $g(\pi)=1$, $t_{j}(\pi)=(1+\lambda)(m-p) \mathcal{s}^{\mathcal{L}}, \forall j \in[0,1]$ and

$$
s_{j}(\pi)= \begin{cases}\hat{s} & \text { for } j=0 \\ 0 & \text { for } j \in] 0, p] \\ s^{\mathcal{L}}(\pi) & \text { for } j \in] p, m] \\ 0 & \text { for } j \in] m, 1]\end{cases}
$$

where $s^{\mathcal{L}}(\pi)=\frac{(1+\lambda) k-V_{l}}{1-(1+\lambda)(m-p)}$.

To prove the claim, note first that proposal $\pi$ will be adopted in equilibrium (i.e. $I(\pi)=1)$ because $u_{j}(\pi) \geq 0, \forall j \in[0, p]$ and $\left.\left.U_{j}(\pi)=0, j \in\right] p, m\right]$.

Second, any other proposal $\pi^{\prime}$ with $S\left(\pi^{\prime}\right)<S(\pi)$ would not be adopted. This follows directly from the fact that a smaller $S(\pi)$ implies that either the fraction of subsidized project losers is smaller than $m-p$ or the subsidy given to each subsidized project loser is smaller than $s^{\mathcal{L}}(\pi)$, or both. However, the fraction of voters supporting $\pi^{\prime}$ is smaller than $m$ and thus $I\left(\pi^{\prime}\right)=0$.

Third, there is no other proposal $\pi^{\prime}$ with $S\left(\pi^{\prime}\right) \geq S(\pi)$ that yields higher utility for the agenda-setter.

From these considerations it follows that proposal $\pi$ maximizes $U_{0}(\pi)=u_{0}(\pi) I(\pi)$ under the restriction that $g(\pi)=1$ and $p<m, V_{w} \geq V_{w}^{*}$.

Step 7: For the agenda-setter, proposal $\pi$ is preferable to proposal $\tilde{\pi}$ as

$$
U_{0}(\tilde{\pi})=\hat{s} \leq \hat{s}+V_{w}-V_{w}^{*}=U_{0}(\pi) .
$$

Hence, in case $p<m$ and $V_{w} \geq V_{w}^{*}$, proposal $\pi$ will be implemented.

Step 8: Consider the case $p<m<\frac{1}{1+\lambda}$ and $V_{w}<V_{w}^{*}$.

We claim that the optimal proposal for the agenda-setter is given by $g(\pi)=1$, $t_{j}=(1+\lambda)\left[p s^{\mathcal{W}}(\pi)+(m-p) s^{\mathcal{L}}(\pi)\right], \forall j \in[0,1]$ and

$$
s_{j}(\pi)= \begin{cases}\hat{s} & \text { for } j=0 \\ s^{\mathcal{W}}(\pi) & \text { for } j \in] 0, p] \\ s^{\mathcal{L}}(\pi) & \text { for } j \in] p, m] \\ 0 & \text { for } j \in] m, 1]\end{cases}
$$


where

$$
\begin{gathered}
s^{\mathcal{W}}(\pi)=\frac{1-(1+\lambda)(m-p)}{1-(1+\lambda) m}\left(V_{w}^{*}-V_{w}\right), \\
s^{\mathcal{L}}(\pi)=\frac{(1+\lambda) k-(1+\lambda) p V_{w}-(1-(1+\lambda) p) V_{l}}{1-(1+\lambda) m} .
\end{gathered}
$$

To prove the claim, we first argue that proposal $\pi$ will be adopted in equilibrium (i.e. $I(\pi)=1$ ), as $\left.\left.u_{j}(\pi)=0, \forall j \in\right] 0, m\right]$.

Second, any other proposal $\pi^{\prime}$ with $S\left(\pi^{\prime}\right)<S(\pi)$ would not be adopted. The reasons are the same as in Step 6.

Third, there is no other proposal $\pi^{\prime}$ with $S\left(\pi^{\prime}\right) \geq S(\pi)$ that yields higher utility to the agenda-setter.

Again, we can conclude that proposal $\pi$ as stated above maximizes $U_{0}(\pi)=$ $u_{0}(\pi) I(\pi)$ under the restriction that $g(\pi)=1$ and $p<m, V_{w}<V_{w}^{*}$.

Step 9: For the agenda-setter, proposal $\tilde{\pi}$ is preferable to proposal $\pi$, as

$$
U_{0}(\tilde{\pi})=\hat{s}>\hat{s}+V_{w}-V_{w}^{*}>U_{0}(\pi) .
$$

Hence, in the case $p<m<\frac{1}{1+\lambda}$ and $V_{w}<V_{w}^{*}$, proposal $\tilde{\pi}$ will be implemented.

Step 10. Consider finally the last case $\max \left\{p, \frac{1}{1+\lambda}\right\}<m$ and $V_{w}<V_{w}^{*}$.

We claim that if $\frac{1}{1+\lambda}<m$ and $V_{w}<V_{w}^{*}$, there is no constitutional proposal for $I(\pi)=1$.

From Step 8 we know that if $V_{w}<V_{w}^{*}$, it will be necessary to subsidize not only a fraction of $m-p$ project losers but also all project winners, i.e. $s^{\mathcal{W}}(\pi)>0$. This is due to the fact that $V_{w}$ is not high enough to compensate project winners for the utility loss incurred by $\operatorname{tax} V_{w}^{*}$. In this case, the overall fraction of subsidized voters is equal to $m$, so the costs for increasing all subsidies by one dollar are equal to $(1+\lambda) m$ (i.e. in order to increase subsidies by one dollar, taxes to the tune of $(1+\lambda) m$ have to be paid). Otherwise the benefit from receiving one dollar of redistribution is equal to one. As $(1+\lambda) m>1$, the costs of redistribution are higher than the benefit from redistribution, so project losers cannot be compensated for their utility loss. ${ }^{15}$

\footnotetext{
${ }^{15}$ In order to compensate project losers for utility losses incurred by $g(\pi)=1$, subsidies should
} 


\section{B Proof of Lemma 6}

We show that an $\varepsilon>0$ exists such that $V_{w}<V_{w}^{*}$ holds for all $\left|V_{w}-k(1+\lambda)\right|<$ $\left|V_{l}-k(1+\lambda)\right|$ and $p<\varepsilon$. We note that

$$
\begin{aligned}
V_{w}^{*}-V_{w} & =(1+\lambda) \frac{k-(m-p) V_{l}}{1-(1+\lambda)(m-p)}-V_{w} \\
& =\frac{(1+\lambda) k-(1+\lambda)(m-p) V_{l}-V_{w}+(1+\lambda)(m-p) V_{w}}{1-(1+\lambda)(m-p)} \\
& >\frac{(1+\lambda) k+(1+\lambda)(m-p)\left(V_{w}-2 k(1+\lambda)\right)-V_{w}+(1+\lambda)(m-p) V_{w}}{1-(1+\lambda)(m-p)} \\
& >\frac{(2(1+\lambda)(m-p)-1)\left(V_{w}-k(1+\lambda)\right)}{1-(1+\lambda)(m-p)},
\end{aligned}
$$

where we have used $-V_{l}>V_{w}-2 k(1+\lambda)$. Recall that for all projects $V_{w}-k(1+\lambda)>0$ holds. Moreover, for all $p<\frac{1}{2} \frac{\lambda}{(1+\lambda)}$ we have $2(1+\lambda)(m-p)-1>0$. Hence PALP holds for constitution $\Pi_{T}$.

\section{Derivation of Welfare}

The utilitarian welfare measure for a particular proposal is given by

$$
W(\pi):=I(\pi)\left[\left(p V_{w}+(1-p) V_{l}-(1+\lambda) k\right) g(\pi)-\lambda S(\pi)\right]
$$

If proposals are not unique, only upper and lower bounds for welfare may be computed. By Definition 1, a redistribution-efficient proposal yields maximal welfare as deadweight loss from redistribution is minimized for all $\pi$ for which $g(\pi) I(\pi)=$ const.

\section{Constitution $\Pi$}

(I.) Highest levels of welfare

(i.) $p \geq m$ :

The lowest level of $S(\pi)$ for $I(\pi)=1$ is given by $S(\pi)=0$. Note that, for this case, the tax scheme must be chosen such that $V_{w}-t_{j} \geq 0$ holds.

become negative (note that $s^{\mathcal{L}}(\pi)$ in Step 8 turns negative if $\left.1-(1+\lambda) m<0\right)$. But as we do not allow for negative subsidies, there is no way to compensate project losers. 
Otherwise project winners would not support the proposal, and the required majority cannot be achieved.

Hence the highest level of welfare under constitution $\Pi$ in case $p \geq m$ is given by

$$
W=p V_{w}+(1-p) V_{l}-(1+\lambda) k
$$

(ii.) $p<m$ :

$S(\pi)$ is minimized if the smallest share of voters is subsidized with the smallest amount of subsidies such that $I(\pi)=1$. The smallest share of subsidized voters occurs if a fraction of $(m-p)$ project losers is subsidized. The minimal subsidy that must be given to them is $\max \left\{0,-V_{l}\right\}$. Again, the tax scheme must be such that project winners and subsidized project losers will support the proposal.

The highest level of welfare under constitution $\Pi$ in case $p<m$ is given by

$$
W=p V_{w}+(1-p) V_{l}-(1+\lambda) k-\lambda(m-p) \max \left\{0,-V_{l}\right\}
$$

(II.) Lowest levels of welfare

No matter if $p \geq m$ or $p<m$, the lowest level of welfare occurs if every voter receives the maximal subsidy $\hat{S}$, i.e. $S=\hat{s}$. Hence the lower bound on welfare is given by

$$
W=p V_{w}+(1-p) V_{l}-(1+\lambda) k-\lambda \hat{s}
$$

\section{Constitutions $\Pi_{\mathrm{T}}, \Pi_{\mathrm{S}}$ and $\Pi_{\mathrm{ST}}$}

Under constitutions $\Pi_{T}, \Pi_{S}$, and $\Pi_{S T}$, the total amount of subsidies $S(\pi)$ is uniquely given and hence welfare functions can be derived directly from Propositions 2-4.

- From Proposition 2

$$
S\left(\pi_{T}^{*}\right)= \begin{cases}(m-p)\left(V_{w}^{*}-V_{w}\right) & \text { if } p<m \text { and } V_{w} \geq V_{w}^{*} \\ 0 & \text { otherwise }\end{cases}
$$

The project will be proposed and implemented if $p<m$ and $V_{w} \geq V_{w}^{*}$ or if $p \geq m$.

Hence the welfare level under constitution $\Pi_{T}$ is given by 


$$
\begin{aligned}
& W\left(\pi_{T}^{*}\right) \\
& = \begin{cases}p V_{w}+(1-p) V_{l}-(1+\lambda) k-\lambda(m-p)\left(V_{w}^{*}-V_{w}\right) & \text { if } p<m \text { and } V_{w} \geq V_{w}^{*} \\
0 & \text { if } p<m \text { and } V_{w}<V_{w}^{*} \\
p V_{w}+(1-p) V_{l}-(1+\lambda) k & \text { if } p \geq m\end{cases}
\end{aligned}
$$

- From Proposition 3

$S(\pi)=\hat{s}$ and the project will always be proposed and adopted. Welfare is given by

$$
W\left(\pi_{S}^{*}\right)=p V_{w}-(1-p) V_{l}-(1+\lambda) k-\lambda \hat{s} .
$$

- From Proposition 4

$S(\pi)=0$ and the project will be adopted only if $p \geq m$. Hence the welfare level under constitution $\Pi_{S T}$ is given by

$$
W\left(\pi_{S T}\right)^{*}= \begin{cases}0 & \text { if } p<m \\ p V_{w}+(1-p) V_{l}-(1+\lambda) k & \text { if } p \geq m\end{cases}
$$

\section{Proof of Proposition 6}

First we note that $p<1-m$ implies $p<m$. Recall that under constitution $\Pi_{S T}$ the project will never be implemented if $p<m$ (see Proposition 4). Hence from an ex-ante perspective all citizens obtain a utility of zero under constitution $\Pi_{S T}$.

Under constitution $\Pi_{T}$ the project may be implemented if $p<m$. More precisely, if $p<m$, the project will be implemented if and only if $V_{w} \geq V_{w}^{*}$ (see Proposition 2). Rewriting this conditions shows that the project will be implemented if and only if

$$
k \leq \frac{1-(1+\lambda)(m-p)}{1+\lambda} V_{w}=: k^{*} .
$$

Hence a citizen's expected utility in the constitutional stage is given by

$$
\mathbb{E}\left[W\left(\pi_{T}^{*}\right)\right]=\frac{1}{\bar{k}} \int_{0}^{\min \left\{\bar{k}, k^{*}\right\}} p V_{w}-(1+\lambda) k-\lambda(m-p) \frac{(1+\lambda) k}{1-(1+\lambda)(m-p)} d k,
$$

where we have used the facts that $k$ is uniformly distributed on $[0 ; \bar{k}]$ and that welfare would be zero for realizations of $k$ with $k>k^{*}$. Equation (24) can be transformed into

$$
\mathbb{E}\left[W\left(\pi_{T}^{*}\right)\right]=\frac{1}{\bar{k}}\left[p V_{w} \min \left\{\bar{k}, k^{*}\right\}-\frac{1}{2}(1+\lambda) \frac{1-(m-p)}{1-(1+\lambda)(m-p)}\left(\min \left\{\bar{k}, k^{*}\right\}\right)^{2}\right] .
$$


Citizens weakly prefer constitution $\Pi_{T}$ over constitution $\Pi_{S T}$ if and only if $\mathbb{E}\left[W\left(\pi_{T}^{*}\right)\right] \geq$ 0 , which is equivalent to

$$
\min \left\{\bar{k}, k^{*}\right\} \leq \frac{2 p(1-(1+\lambda)(m-p)}{(1+\lambda)(1-(m-p))} V_{w}=: \hat{k}
$$

It is straightforward to show that $k^{*}>\hat{k}$ for $1-p>m$. As a consequence, utilitarian welfare is higher for $\Pi_{T}$ if $\bar{k}<\hat{k}$. It is higher for $\Pi_{S T}$ if $\bar{k}>\hat{k}$.

\section{E $\quad$ Proof of Proposition 7}

In order to examine the agenda-setter's incentives for improving the project, it will be useful to consider her utility for given project parameters and for each constitution. From Propositions 1 to 4 we obtain

$$
\begin{aligned}
& U_{0}\left(\pi^{*}\right)=\hat{s}+V_{w} \\
& U_{0}\left(\pi_{T}^{*}\right)= \begin{cases}\hat{s}+V_{w}-V_{w}^{*} & \text { if } p<m \text { and } V_{w} \geq V_{w}^{*} \\
\hat{s} & \text { if } p<m \text { and } V_{w}<V_{w}^{*} \\
\hat{s}+V_{w}-(1+\lambda) k & \text { if } p \geq m .\end{cases} \\
& U_{0}\left(\pi_{S}^{*}\right)=\hat{s}+V_{w} \text { if } p<m \\
& U_{0}\left(\pi_{S T}^{*}\right)= \begin{cases}0 & \text { if } p \geq m . \\
V_{w}-(1+\lambda) k\end{cases}
\end{aligned}
$$

Constitutions involving an arbitrary tax code (i.e. constitutions $\Pi$ and $\Pi_{S}$ ) yield utility to the agenda-setter that is independent of the project parameters $V_{l}, k$, and $p$. Hence exerting costly effort to enhance any project parameter other than $V_{w}$ will never be profitable.

Under constitution $\Pi_{S T}$ the agenda-setter may profit from exerting effort if $p$ can be increased from $\underline{p}<m$ to $\bar{p} \geq m$. For sufficiently small costs $c$, exerting effort in order to reduce $k$ is optimal for $p \geq m$.

Under constitution $\Pi_{T}$ the agenda-setter profits from increasing $p$ from $\underline{p}<m$ to $\bar{p} \geq m$ if $c$ is sufficiently small. Moreover, the agenda-setter has an incentive to increase $p$ even in the case $p<m$, as long as $V_{w} \geq V_{w}^{*}$. If $p<m$ and $V_{w}<V_{w}^{*}$, the agenda-setter 
has no incentive to enhance project efficiency. If $p \geq m$, the agenda-setter may have incentives to reduce project costs $k$ as under constitution $\pi_{S T}$. If $p<m$ and $V_{w} \geq V_{w}^{*}$, the agenda-setter has incentives to increase $V_{l}$ and to reduce $k$ (as $V_{w}^{*}$ is decreasing in $V_{l}$ and increasing in $k$ ).

Of course, the agenda-setter will enhance project efficiency if and only if the net gains from exerting effort exceed the costs involved in the effort. 


\section{References}

Philippe Aghion and Patrick Bolton. Incomplete Social Contracts. Journal of the European Economic Association, 1(1):38-67, March 2003.

Charles L. Ballard, John B. Shoven, and John Whalley. General Equilibrium Computations of the Marginal Welfare Costs of Taxes in the United States. American Economic Review, 75(1):128-138, 1985.

Felix Bierbrauer. On the Legitimacy of Coercion for the Financing of Public Goods. Max Planck Institute, Working Paper, 2009.

Edgar K. Browning. On the Marginal Welfare Cost of Taxation. American Economic Review, 77(1):11-23, 1987.

James M. Buchanan and Gordon Tullock. The Calculus of Consent. University of Michigan Press, Ann Arbor, 1962.

Eddie Dekel, Matthew O. Jackson, and Asher Wolinsky. Vote Buying: General Elections. Journal of Political Economy, 116(2):351-380, 2008.

Hans Gersbach. Fiscal Constitutions. Constitutional Political Economy, 15(1):3-25, March 2004.

Hans Gersbach. Democratic Mechanisms. Journal of the European Economic Association, 2009. forthcoming.

Tim Groseclose and James M. Snyder. Buying Supermajorities. The American Political Science Review, 90(2):303-315, 1996.

Werner Güth and Martin F. Hellwig. The Private Supply of a Public Good. Journal of Economics/Zeitschrift für Nationalökonomie, 5:121-159, 1986.

Martin F. Hellwig. Public-Good Provision with many Participants. Review of Economic Studies, 70:589-614, 2003.

Martin F. Hellwig. Optimal Income Taxation, Public-Goods Provision and PublicSector Pricing: A Contribution to the Foundations of Public Economics, 2004. mimeo. 
Matthew O. Jackson. A Crash Course in Implementation Theory. Social Choice and Welfare, 18(4):655-708, October 2001.

Alessandro Lizzeri and Nicola Persico. The Provision of Public Goods under Alternative Electoral Incentives. American Economic Review, 91(1):225-239, 2001.

George J. Mailath and Andrew Postlewaite. Asymmetric Information Bargaining Procedures with Many Agents. Review of Economic Studies, 57:351-367, 1990.

Douglas W. Rae. Decision Rules and Individual Values in Constitutional Choice. American Science Review, 63:40-56, 1969.

John Rawls. A Theory of Justice. Harvard University Press, Cambridge, 1971.

Rafael Rob. Pollution Claim Settlements Under Private Information. Journal of Economic Theory, 47:307-333, 1989.

Thomas Romer and Howard Rosenthal. A Constitution for Solving Asymmetric Externality Games. American Journal of Political Science, 27:1-26, 1983.

Charles Stuart. Welfare Costs per Dollar of Additional Tax Revenue in the United States. American Economic Review, 74(3):352-362, 1984.

Michael J. Taylor. Proof of a Theorem on Majority Rule. Behavioral Science, 14: 228-231, 1969. 


\section{Working Papers of the Center of Economic Research at ETH Zurich}

(PDF-files of the Working Papers can be downloaded at www.cer.ethz.ch/research).

09/109 H. Gersbach, V. Hahn and S. Imhof

Constitutional Design: Separation of Financing and Project Decision

09/108 C. N. Brunnschweiler

Oil and Growth in Transition Countries

09/107 H. Gersbach and V. Hahn

Banking-on-the-Average Rules

09/106 K. Pittel and D.T.G. Rübbelke

Decision Processes of a Suicide Bomber - Integrating Economics and Psychology

08/105 A. Ziegler, T. Busch and V.H. Hoffmann

Corporate Responses to Climate Change and Financial Performance: The Impact of Climate Policy

09/104 S. Valente

Endogenous Growth, Backstop Technology Adoption and Optimal Jumps

09/103 K. Pittel and D. Rübbelke

Characteristics of Terrorism

09/102 J. Daubanes

Taxation of Oil Products and GDP Dynamics of Oil-rich Countries

09/101 S. Valente

Accumulation Regimes in Dynastic Economies with Resource Dependence and Habit Formation

08/100 A. Ziegler

Disentangling Specific Subsets of Innovations: A Micro-Econometric Analysis of their Determinants

08/99 M. Bambi and A. Saïdi

Increasing Returns to Scale and Welfare: Ranking the Multiple Deterministic Equilibria

08/98 M. Bambi

Unifying time-to-build theory

08/97 H. Gersbach and R. Winkler

International Emission Permit Markets with Refunding

08/96 K. Pittel and L. Bretschger

Sectoral Heterogeneity, Resource Depletion, and Directed Technical Change: Theory and Policy 
08/95 M. D. König, S. Battiston, M. Napoletano and F. Schweitzer The Efficiency and Evolution of R\&D Networks

08/94 H. Gersbach and F. Mühe Vote-Buying and Growth

08/93 H. Gersbach Banking with Contingent Contracts, Macroeconomic Risks, and Banking Crises

08/92 J. Daubanes Optimal taxation of a monopolistic extractor: Are subsidies necessary?

08/91 R. Winkler Optimal control of pollutants with delayed stock accumulation

08/90 S. Rausch and T. F. Rutherford Computation of Equilibria in OLG Models with Many Heterogeneous Households

08/89 E. J. Balistreri, R. H. Hillberry and T. F. Rutherford Structural Estimation and Solution of International TradeModels with Heterogeneous Firms

08/88 E. Mayer and O. Grimm

Countercyclical Taxation and Price Dispersion

08/87 L. Bretschger Population growth and natural resource scarcity: long-run development under seemingly unfavourable conditions

08/86 M. J. Baker, C. N. Brunnschweiler, and E. H. Bulte Did History Breed Inequality? Colonial Factor Endowments and Modern Income Distribution

08/85 U. von Arx and A. Ziegler The Effect of CSR on Stock Performance: New Evidence for the USA and Europe

08/84 H. Gersbach and V. Hahn Forward Guidance for Monetary Policy: Is It Desirable?

08/83 I. A. MacKenzie On the Sequential Choice of Tradable Permit Allocations

08/82 I. A. MacKenzie, N. Hanley and T. Kornienko A Permit Allocation Contest for a Tradable Pollution Permit Market

08/81 D. Schiess and R. Wehrli The Calm Before the Storm? - Anticipating the Arrival of General Purpose Technologies

08/80 D. S. Damianov and J. G. Becker Auctions with Variable Supply: Uniform Price versus Discriminatory 
08/79 H. Gersbach, M. T. Schneider and O. Schneller

On the Design of Basic-Research Policy

08/78 C. N. Brunnschweiler and E. H. Bulte

Natural Resources and Violent Conflict: Resource Abundance, Dependence and the Onset of Civil Wars

07/77 A. Schäfer, S. Valente

Habit Formation, Dynastic Altruism, and Population Dynamics

07/76 R. Winkler

Why do ICDPs fail? The relationship between subsistence farming, poaching and ecotourism in wildlife and habitat conservation

$07 / 75$ S. Valente

International Status Seeking, Trade, and Growth Leadership

07/74 J. Durieu, H. Haller, N. Querou and P. Solal

Ordinal Games

07/73 V. Hahn

Information Acquisition by Price-Setters and Monetary Policy

07/72 H. Gersbach and H. Haller

Hierarchical Trade and Endogenous Price Distortions

07/71 C. Heinzel and R. Winkler

The Role of Environmental and Technology Policies in the Transition to a Lowcarbon Energy Industry

07/70 T. Fahrenberger and H. Gersbach

Minority Voting and Long-term Decisions

07/69 H. Gersbach and R. Winkler

On the Design of Global Refunding and Climate Change

$07 / 68$ S. Valente

Human Capital, Resource Constraints and Intergenerational Fairness

07/67 O. Grimm and S. Ried

Macroeconomic Policy in a Heterogeneous Monetary Union

07/66 O. Grimm

Fiscal Discipline and Stability under Currency Board Systems

07/65 M. T. Schneider

Knowledge Codification and Endogenous Growth

07/64 T. Fahrenberger and H. Gersbach

Legislative Process with Open Rules

07/63 U. von Arx and A. Schäfer

The Influence of Pension Funds on Corporate Governance 